\title{
Characteristic time of thermal and diffusional relaxation
}

\author{
(C) O. V. Aryasova, Ya. M. Khazan, 2015
}

\author{
Institute of Geophysics, National Academy of Sciences of Ukraine, Kiev, Ukraine \\ Received October 12, 2015 \\ Presented by the Editorial Board Member V. N. Shuman
}

\begin{abstract}
Показано, что часто используемая размерная оценка времени тепловой релаксации $t_{r}=L^{2} / \kappa$, где $L-$ мощность слоя, а к- температуропроводность, дает сильно завышенное значение $t_{r}$. Правильная оценка $t_{r}=L^{2} / \pi^{2} \kappa$ должна содержать множитель $1 / \pi^{2}$ порядка 0,1.

Ключевые слова: характерное время, тепловая релаксация.
\end{abstract}

Introduction. Notwithstanding the correct way to estimate thermal and diffusional relaxation time is well known and straightforward, in some publications estimates of thermal relaxation time contain major errors. Most typically, when estimating the thermal relaxation time based upon dimensional analysis, $t_{r}=C L^{2} / \kappa$, where $L$ is a layer thickness and $\kappa$ is heat diffusivity, one assumes by default that the numerical factor, $C$, is of the order of unity while for a 1D geometry it is, in fact, $1 / \pi^{2}$. As a consequence, a statement that a characteristic thermal relaxation time for a lithosphere of, say, $L=200 \mathrm{~km}$ thickness is of the order of a billion years is incorrect. The same relates to an estimate of time necessary for a thermal perturbation at a depth $L$ to manifest itself in the surface heat flow. Actually, in both cases the characteristic time is of $L^{2} / \pi^{2} \kappa$, i. e. as short as ca. 140 m.y. for $L=200 \mathrm{~km}$ and $\kappa=10^{-6} \mathrm{~m}^{2} \mathrm{~s}^{-1}$.

In the present letter we remind at the beginning what is meant by the term «the relaxation time», and then discuss a few most common problems where this value is useful. To be specific, in what follows we speak of the thermal relaxation although all results up to notation are valid for the problem of the diffusional relaxation.

For those who are not interested in the mathematical details, Table 1 summarizes the relaxation time expressions for a number of boundary conditions and the geometry of the system (one-dimensional or spherically symmetric). Also, Table 2 lists the thermal relaxation time estimates for the continental (Precambrian) and oceanic lithosphere. The estimates assume $\kappa=0.8 \cdot 10^{-6} \mathrm{~m}^{2} \mathrm{~s}^{-1}$. The values of Archaean and Proterozoic lithosphere thicknesses are from [Artemieva, 2009]. In the suboceanic mantle, a thickness of the conductive lithosphere as well as a thickness of the thermal boundary layer (the lithosphere together with a layer accom- modating a transition to the convecting mantle) depends on rock rheology and potential temperature, $T_{p}$, of the mantle convection [Khazan, Aryasova, 2014]. The values in Table 2 are based upon the laboratory data by Hirth and Kohlstedt [2003] and $T_{p}$ range of $1350^{\circ} \mathrm{C}$ to $1300^{\circ} \mathrm{C}$ (see details in [Khazan, Aryasova, 2014]).

It is instructive to observe in Figs. 1 and 2 how a layer thermally perturbed at its base approaches a new steady state.

The definition of relaxation time. Let a dependence of a system on time, $t$, be characterized by a function $A(t)$ with the value $A=A_{0}$ of the function corresponding to the stable equilibrium. The latter means that if the system is brought out of the equilibrium then the sign of the rate $d A / d t$ is opposite to that of the deviation $A-A_{0}$ and $d A / d t$ vanishes at $A=A_{0}$. Therefore, the series expansion of $d A / d t$ contains only odd powers of $A-A_{0}$. In the simplest case only linear term remains:

$$
\frac{d A}{d t}=-\gamma\left(A-A_{0}\right),
$$

where $\gamma$ is a positive factor.

The solution to Eq. (1) is as follows

$$
A(t)=A_{0}+\left(A_{1}-A_{0}\right) e^{-\gamma t},
$$

where $A_{1}$ is the initial value of $A$, i. e. $A(0)=A_{1}$.

One can see from Eq. (2) that the absolute value of the system deviation from the equilibrium, $\mid A(t)-$ $A_{0} \mid$, decreases exponentially, and the time required for $\left|A(t)-A_{0}\right|$ to decay from its initial value $\left|A_{1}-A_{0}\right|$ to $1 / e$ of that value is $t_{r}=1 / \gamma$. This time interval is commonly referred to as the relaxation time. Note that the relaxation time is independent of the initial deviation.

Thermal relaxation time for an infinite homogeneous layer with fixed temperature at the 


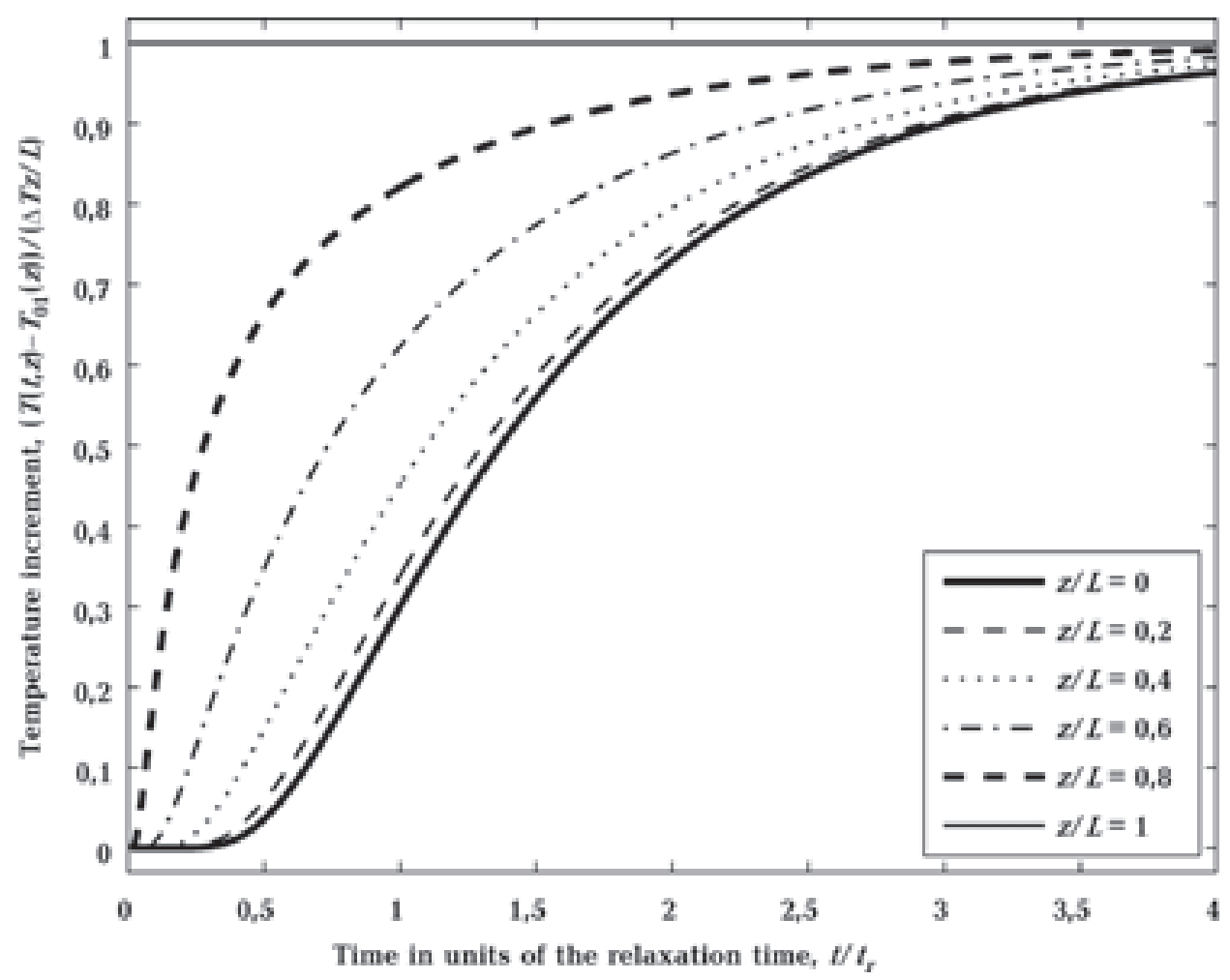

Fig. 1. The temperature increment (Eq. (14); the term in square brackets) vs. time in units of the characteristic relaxation time (Eq. (13)).

Ta b l e 1. Thermal/diffusional relaxation time, $t_{r}$, depending on the geometry and the boundary conditions of the problem ( $\kappa$ is thermal diffusivity, $D$ is diffusion coefficient)

\begin{tabular}{|l|l|c|}
\hline \multicolumn{1}{|c|}{ Problem } & \multicolumn{1}{|c|}{ Boundary conditions } & Relaxation time \\
\hline $\begin{array}{l}\text { Relaxation of the temperature in } \\
\text { a layer of thickness } L \text { after an in- } \\
\text { stantaneous change of basal tem- } \\
\text { perature. }\end{array}$ & $\begin{array}{l}\text { Temperature boundary condition on both } \\
\text { boundaries }\end{array}$ & $t_{r}=\frac{L^{2}}{\pi^{2} \kappa}$ \\
\hline $\begin{array}{l}\text { Time necessary for a thermal } \\
\text { perturbation at a depth } L \text { to mani- } \\
\text { fest itself in the surface heat flow }\end{array}$ & The same & $t_{r}=\frac{L^{2}}{\pi^{2} \kappa}$ \\
\hline $\begin{array}{l}\text { Relaxation of the temperature in a } \\
\text { layer after an instantaneous change } \\
\text { in heat flow through its base }\end{array}$ & $\begin{array}{l}\text { Temperature and heat flow boundary con- } \\
\text { ditions on top and bottom boundary, res- } \\
\text { pectively }\end{array}$ & $t_{r}=\frac{4 L^{2}}{\pi^{2} \kappa}$ \\
\hline $\begin{array}{l}\text { Relaxation of the temperature (mi- } \\
\text { nor element abundance) in a sphe- } \\
\text { rical body }\end{array}$ & $\begin{array}{l}\text { Zero temperature (zero abundance) boun- } \\
\text { dary condition. In the center of the body } \\
\text { the temperature (abundance) is finite. }\end{array}$ & $t_{r}=\frac{R^{2}}{\pi^{2} \kappa}$, \\
\hline
\end{tabular}

boundaries. To be specific, we assume that the layer is horizontal, the axis $z$ is directed downward, and the temperature at the surface is zero.

The heat propagation in the layer is described by the 1D heat equation:

$$
\frac{\partial T}{\partial t}=\kappa \frac{\partial^{2} T}{\partial z^{2}}+Q(z)
$$

where $T(t, z)$ is temperature, $t$ is time, $z$ is depth, $\kappa$ is the heat diffusivity, and $Q(z)$ is to account for the heat generation. 


\section{Ta b l e 2. Some useful estimates of the relaxation time}

\begin{tabular}{|l|c|c|c|}
\hline & Thickness, km & Age, Ma & $t_{r^{\prime}}$ Ma \\
\hline $\begin{array}{l}\text { Continental lithosphere } \\
\text { Archaean } \\
\text { Proterozoic }\end{array}$ & $\begin{array}{c}180-250 \\
150-180\end{array}$ & $\begin{array}{c}>2500 \\
500-2500\end{array}$ & $\begin{array}{c}137-264 \\
95-137\end{array}$ \\
\hline $\begin{array}{l}\text { Suboceanic mantle } \\
\text { Lithosphere } \\
\text { Thermal boundary layer }\end{array}$ & $50-70^{*}$ & $<200$ & $11-21$ \\
& $75-110^{*}$ & & $25-49$ \\
\hline
\end{tabular}

* The range of thicknesses corresponds to the range of values of the mantle convection potential temperature of $1350{ }^{\circ} \mathrm{C}$ to $1300^{\circ} \mathrm{C}$ (see text).

Assume that at $t<0$ the layer was in its steady state $T_{01}(z)$, satisfying the steady state version of Eq. (3)

$$
\kappa \frac{\partial^{2} T}{\partial z^{2}}+Q(z)=0
$$

with the basal temperature $T(L)=T_{1}$.

At $t=0$ the basal temperature changes stepwise from $T_{1}$ to $T_{2}=T_{1}+\Delta T$ and the layer relaxes to the new steady state

$$
T_{02}(z)=T_{01}(z)+\Delta T \frac{z}{L},
$$

satisfying Eq. (4) with the basal temperature $T_{02}(L)=T_{2}$.

The solution $T(t, z)$ to Eq. (3) with the boundary conditions $T(t, 0)=0, T(t, L)=T_{2}$ and the initial condition $T(0, z)=T_{01}(z)$ may be expressed as

$$
T(t, z)=T_{02}(z)-u(t, z),
$$

where $u(t, z)=T_{02}(z)-T(t, z)$ is the difference between the steady state temperature, $T_{02}(z)$, and the current one, $T(t, z) .|u(t, z)|$ is maximum at $t=0$ and tends to zero with time. $u(t, z)$ is the solution to Eq. (3) without sources

$$
\frac{\partial u}{\partial t}=\kappa \frac{\partial^{2} u}{\partial z^{2}}
$$

with zero boundary conditions $u(t, 0)=u(t, L)=0$ and the initial condition $u(0, z)=T_{02}(z)-T_{01}(z)=\Delta T z / L$. In terms of dimensionless coordinate $\zeta=z / L, 0 \leq \zeta \leq 1$, and time $\tau=t \kappa / L^{2}$ the boundary value problem Eq. (7) for function $w(\tau, \zeta)=T / \Delta T$ may be rewritten as

$$
\frac{\partial w}{\partial t}=\frac{\partial^{2} w}{\partial \zeta^{2}}, w(\tau, 0)=w(\tau, 1)=0, w(0, \zeta)=\zeta .
$$

To apply the standard method of separation of variables we make the substitution of the form $w(\tau, \zeta)=\mathrm{T}(\tau) Z(\zeta)$ and for the space part $Z(\zeta)$ obtain an eigenvalue problem:

$$
Z^{\prime \prime}+\lambda Z=0, Z(0)=Z(1)=0,
$$

where $\lambda$ is the separation parameter.

A general solution of Eq. (9) satisfying the boundary conditions is

$$
\mathrm{Z}_{n}(\zeta)=C_{n} \sin \sqrt{\lambda_{n}} \zeta, \lambda_{n}=\pi^{2} n^{2}, n=1,2, \ldots
$$

Solving also the equation for the time part $\mathrm{T}(\tau)$ one may write a general solution to problem Eq. (8) as an exponentially converging series:

$$
w(\tau, \zeta)=\sum_{n=1}^{\infty} C_{n} e^{-\pi^{2} n^{2} \tau} \sin \pi n \zeta .
$$

Here $C_{n}=2(-1)^{n+1} / \pi n$ is Fourier coefficients of sine expansion of the initial condition $w(0, \zeta)=\zeta$.

Since the series (11) converges exponentially, the dimensionless relaxation time may be estimated based upon its first term:

$$
\tau_{r}=\frac{1}{\lambda_{1}}=\frac{1}{\pi^{2}} \cong 0,101 .
$$

The dimensional relaxation time is as follows:

$$
t_{r}=\frac{L^{2}}{\pi^{2} \kappa} .
$$

Now, one may estimate from Eq. (13) that the characteristic relaxation time of Archaean lithosphere of $L=200 \mathrm{~km}$ thick is $t_{r}=168 \mathrm{~m}$. y. while for a $40 \mathrm{~km}$ crust $t_{r}=6.8 \mathrm{~m}$. y. (in both estimates the value $\kappa=0.8 \cdot 10^{-6} \mathrm{M}^{2} \mathrm{C}^{-1}$ is used).

The closed dimensional solution to Eq. (3) may be written as:

$$
\begin{gathered}
T(t, z)=T_{01}(z)+ \\
+\Delta T \frac{z}{L}\left[1+2 \frac{L}{z} \sum_{n=1}^{\infty} \frac{(-1)^{n}}{\pi n} \sin \pi n \frac{z}{L} e^{-\pi^{2} n^{2} \frac{\kappa}{L^{2}}}\right] .
\end{gathered}
$$

The second term in the latter equation char- 
acterizes an increment of the temperature due to a thermal perturbation at the bottom of the layer. This term is zero initially and tends to $\Delta T z / L$ afterwards. The most slowly the temperature approaches to its steady state value in the vicinity of the surface, i.e. the farthest from the perturbation source. However, even in the close vicinity of the surface, e. g. at $z / L=0.01$, this term is of $0.3 \Delta T z / L$ at $t=t_{r^{\prime}} 0.73 \Delta T z / L$ at $t=2 t_{r^{\prime}}$ and $0.9 \Delta T z / L$ at $t=3 t_{r}$. The approach of the temperature to the new steady state is illustrated in Fig. 1.

It is also instructive to calculate the variation of the heat flow $q(t, \mathrm{z})=-\kappa \partial T(t, \mathrm{z}) / \partial \mathrm{z}$ due to the thermal perturbation operating at the depth $L$. It is straightforward to see from Eq. (14) that

$$
\begin{gathered}
q(t, z)=q_{01}(z)+ \\
+\Delta q\left[1+2 \sum_{n=1}^{\infty}(-1)^{n} \cos \pi n \frac{z}{L} e^{-\pi^{2} n^{2} \frac{\kappa t}{L^{2}}}\right],
\end{gathered}
$$

where $q_{01}(\mathrm{z})=-\kappa \partial T_{01}(\mathrm{z}) / \partial \mathrm{z}$ is the initial value of the heat flow at the depth $z$, and $\Delta q=-\kappa \Delta T / L$ is the additional steady state heat flow due to the perturbation. The relaxation of the heat flow at different depths is illustrated in Fig. 2. In particular, it follows from Eq. (15) that the characteristic time necessary for the perturbation operating at the depth $L$ to affect the surface heat flow is $t_{r}$. Actually, the surface heat flow increment (the second term in the right hand side of Eq. (15) taken at $z=0$ ) is of $0.3 \Delta q$ at $t=t_{r^{\prime}} 0.7 \Delta q$ at $t=2 t_{r^{\prime}}$ and $0.9 \Delta q$ at $t=3 t_{r^{\prime}}$.

Thermal relaxation time of a homogeneous layer in the case of the heat flow bottom boundary condition. From the geophysical viewpoint, this problem does not make much sense because under natural conditions in the Earth a system in which one part supplies a constant heat flux into another part cannot occur. On the other hand, one can imagine a body irradiated by a beam of particles absorbed by the body surface and diffusing inward. This situation is well described by constant particle flux boundary condition.

The evolution of the system with constant heat flow boundary condition is described by Eq. (3) with different boundary conditions:

$$
\frac{\partial T}{\partial t}=\kappa \frac{\partial^{2} T}{\partial z^{2}}+Q(z) ; T(t, 0)=0 ;-\kappa \frac{\partial T(t, L)}{\partial z}=q_{0} .
$$

Let $T_{01}(z)$ be the steady state solution to Eq. (16), i. e. the solution satisfying Eq. (16) with zero

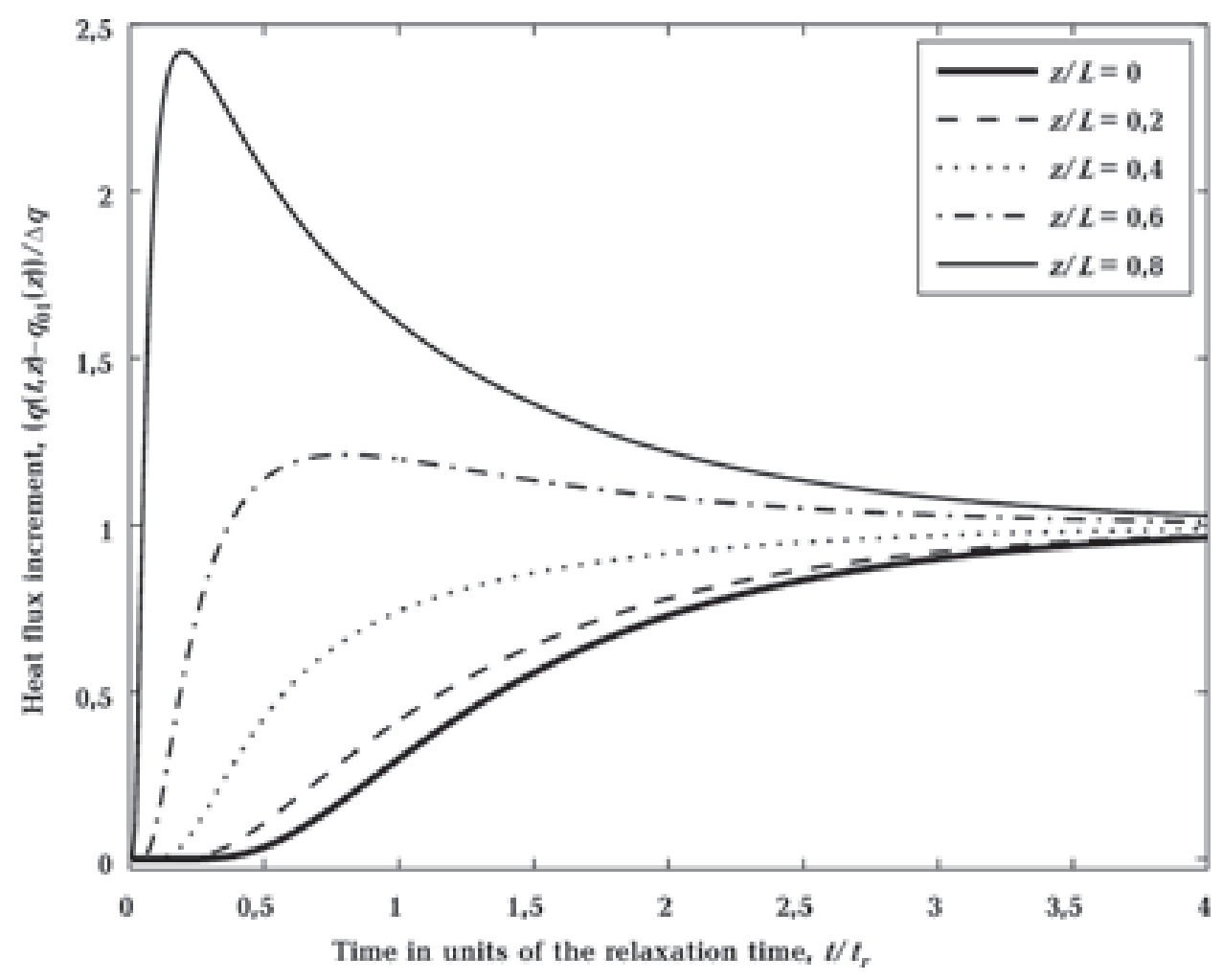

Fig. 2. Increment of the heat flux at different depths (Eq. 15; the term in the square brackets) vs. time in units of the characteristic relaxation time (Eq. (13)). 
left hand side. If at $t=0$ the heat flow through the bottom boundary changes by $\Delta q$, the bottom boundary condition becomes as follows:

$$
-\kappa \frac{\partial u(t, L)}{\partial z}=q_{0}+\Delta q .
$$

After the relaxation is over, the new steady state is

$$
T_{02}(t, z)=T_{01}(z)-\frac{\Delta q z}{\kappa} .
$$

It is convenient to look for the solution to Eq. (16) with boundary condition Eq. (17) in the form

$$
T(t, z)=T_{02}(z)+w(t, z),
$$

where $w(t, z)$ is the solution to Eq. (16) without sources, with zero surface temperature, zero bottom boundary heat flow, and initial condition $w(0, z)=T(0, z)-T_{02}(z)=z \Delta q / \kappa$.

Introducing now dimensionless coordinate $\zeta=z / L$, time $\tau=\kappa t / L^{2}$, and temperature $u=\kappa w / L \Delta q$, we arrive at the problem

$$
\frac{\partial u}{\partial \tau}=\frac{\partial^{2} u}{\partial \zeta^{2}}, u(\tau, 0)=0, \frac{\partial u(\tau, 1)}{\partial \zeta}=0, u(0, \zeta)=\zeta .
$$

Similarly to the preceding problem Eq. (8), this one may be solved using the separation of variables method. The final solution may be written as

$$
\begin{gathered}
T(t, z)=T_{01}(z)-\frac{\Delta q L}{\kappa} \frac{z}{L} \times \\
\times\left[1-\frac{8}{\pi^{2}} \frac{L}{z} \sum_{n=0}^{\infty} \frac{(-1)^{n}}{(2 n+1)^{2}} \sin \pi\left(\frac{1}{2}+n\right) \frac{z}{L} e^{-\pi^{2}\left(\frac{1}{2}+n\right)^{2}} \frac{\kappa t}{L^{2}}\right] .
\end{gathered}
$$

Note that if the heat flows upward then $\Delta q$ is negative. The characteristic relaxation time for this problem is

$$
t_{r}=\frac{4 L^{2}}{\pi^{2} \kappa},
$$

i. e. in a factor of 4 longer than that for the problem Eq. (3) with temperature boundary condition at the bottom boundary. At $t=t_{r}$ the temperature increment (the second term in the right hand side of Eq. (21)) is about 0.6 of the steady state value $|\Delta q| z / \kappa$, at $t=2 t_{r}$ is of $0.85|\Delta q| z / \kappa$, and at $t=3 t_{r}$ is of $0.95|\Delta q| z / \kappa$.

Relaxation time of impurity abundance/temperature in a sphere. Consider a problem of diffusion of an absolutely incompatible impurity from a spherical «crystal» into melt. Since the distribution coefficient for the impurity equals to zero, the boundary condition for the problem is zero impurity abundance. The second boundary condition is the finiteness of the abundance in the sphere center. Up to the notation, the problem is the same as the problem of a sphere cooling.

The governing equation for the diffusion problem is as follows:

$$
\begin{gathered}
\frac{\partial C}{\partial t}=D \frac{1}{r^{2}} \frac{\partial}{\partial r}\left(r^{2} \frac{\partial C}{\partial r}\right), \\
C(t, R)=0, C(t, 0)<\infty, C(0, r)=C_{0}, 0 \leq r<R,
\end{gathered}
$$

where $C(t, r)$ is abundance, $r$ is the distance from the sphere center, $R$ is the sphere radius, $D$ is diffusivity.

Similarly to preceding problems it is useful to introduce dimensionless radius $\rho=r / R$ and time $\tau=D t / R^{2}$ and use the separation of variables method, i. e. look for the solution in the form $C(\tau, \rho)=\mathrm{T}(\tau)$ $\mathrm{P}(\rho)$. As usually, for the space part one obtains the eingenvalue problem

$$
\begin{aligned}
& P^{\prime \prime}+\frac{2}{\rho} P^{\prime}+\lambda P=0, \\
& \mathrm{P}(1)=0, \mathrm{P}(0)<\infty,
\end{aligned}
$$

where $\lambda$ is the separation parameter.

A general solution to Eq.(24), satisfying the condition of the abundance finiteness in the center, is $\mathrm{P}(\rho)=B \rho^{-1 / 2} J_{1 / 2}\left(\lambda^{1 / 2} \rho\right)$, where $B$ is a constant, $J_{1 / 2}(x)$ is the first kind Bessel function of real argument and of order 1/2: $J_{1 / 2}(x)=(2 / \pi x)^{1 / 2} \sin x$. The eigenvalue of the problem is $\lambda_{n}=\pi^{2} n^{2}$, and a general solution to Eq. (23) is:

$$
\frac{C}{C_{0}}=2 \sum_{n=1}^{\infty}(-1)^{n+1} \frac{\sin \pi n r / R}{\pi n r / R} e^{-\pi^{2} n^{2} \frac{D t}{R^{2}}} .
$$

The complete content of the impurity in the "crystal» decreases with time in agreement with the next equation

$$
\frac{\int_{0}^{R} 4 \pi r^{2} C(t, r) d r}{4 \pi R^{3} C_{0} / 3}=6 \sum_{n=1}^{\infty} \frac{1}{\pi^{2} n^{2}} e^{-\pi^{2} n^{2} \frac{D t}{R^{2}}} .
$$

The convergence of the abundance to the steady state zero value is controlled by the slowest varying first term of the series (26). Therefore, the characteristic time of the diffusional relaxation in the sphere of radius $R$ is $t_{r}=R^{2} / \pi^{2} D$. 


\section{Characteristic time of thermal and diffusion relaxation}

\section{(C) O. V. Aryasova, Y. M. Khazan, 2015}

It is shown that frequently used dimensional estimate of a layer thermal relaxation time, $t_{r}$ as $L^{2} / \kappa$ based on the layer thickness, $L$, and thermal diffusivity, $\kappa$, strongly overestimates $t_{r}$. The correct estimate $t_{r}=L^{2} / \pi^{2} \kappa$ should contain a factor of $1 / \pi^{2}$ which is of the order of 0.1 .

Key words: characteristic time, thermal relaxation.

\section{References}

Artemieva I. M., 2009. The continental lithosphere: Reconciling thermal, seismic, and petrologic data. Lithos 109(12), 23-46.

Hirth G., Kohlstedt D. L., 2003. Rheology of the upper mantle and the mantle wedge: A view from the experimentalists. In: Inside the Subduction Factory.
Vol. 138. Geophys. Monogr. Ser., AGU, Washington, D.C. P. $83-105$.

Khazan Ya. M., Aryasova O. V., 2014. Stability of the boundary layer between the lithosphere and convecting mantle and the steady-state lithospheric geotherm. Izvestiya. Physics of the Solid Earth 50(4), 543-561. 